\title{
The effect of flares on total solar irradiance
}

\author{
Matthieu Kretzschmar ${ }^{1 \star}$, Thierry Dudok de Wit ${ }^{1}$, Werner Schmutz ${ }^{2}$, Sabri Mekaoui ${ }^{3}$, \\ Jean-François Hochedez ${ }^{4}$ and Steven Dewitte ${ }^{3}$
}

\begin{abstract}
Flares are powerful bursts of energy released by relatively poorly understood processes that take place in the atmospheres of stars'. However, although solar flares, from our own Sun, are the most energetic events in the solar system, in comparison to the total output of the Sun they are barely noticeable $e^{2,3}$. Consequently, the total amount of radiant energy they generate is not precisely known, and their potential contribution to variations in the total solar irradiance ${ }^{4}$ incident on the Earth has so far been overlooked. In this work, we identify a measurable signal from relatively moderate solar flares in total solar irradiance data. We find that the total energy radiated by flares exceeds by two orders of magnitude the flare energy radiated in the soft-X-ray domain only, indicating a major contribution in the visible domain. These results have implications for our understanding of solar-flare activity and the variability of our star.
\end{abstract}

Flares are sudden bursts of electromagnetic radiation occurring in stellar atmospheres. They are preferentially observed in cool dMe stars where contrast is higher. However, flares have also been observed in other types of star ${ }^{1}$. In the case of the Sun, imaging capabilities have made it possible to observe flares in the visible domain $^{5,6}$ and at short wavelengths, in the X-ray or extremeultraviolet domains, where the contrast is highest and highquality space instrumentation has been purpose built. However, the total energy radiated by flares and its spectral distribution remains largely unknown. In fact, the flare signal in the visible domain or in spectrally integrated observations is hindered by the background fluctuations that are caused both by internal acoustic waves and by solar granulation (see Fig. 1). This explains why, among the more than 20,000 flares that occurred in the most recent solar cycle, only four exceptionally large ones have been identified up to now $^{7}$ in the total solar irradiance (TSI), that is, the flux of solar light received at all wavelengths at the top of the Earth's atmosphere. The TSI is the primary energy input to Earth, so identifying the impact of flares on TSI is of fundamental importance in several aspects: it provides an important constraint for the physics of flares, it makes it possible to compare flares on other stars and it offers an estimate of flare contribution to solar luminosity.

To identify the effect of flares on the TSI, the aforementioned fluctuations must be somehow cancelled out; to do so, we carried out a conditional-average analysis ${ }^{8}$, which is also known as a superposed-epoch analysis. The procedure involves (1) selecting a set of flares, (2) extracting an excerpt of the irradiance time series around the flare peak time for these events and (3) averaging (or superposing) these smaller time series in such a way that flares occur at the same time in all time series.
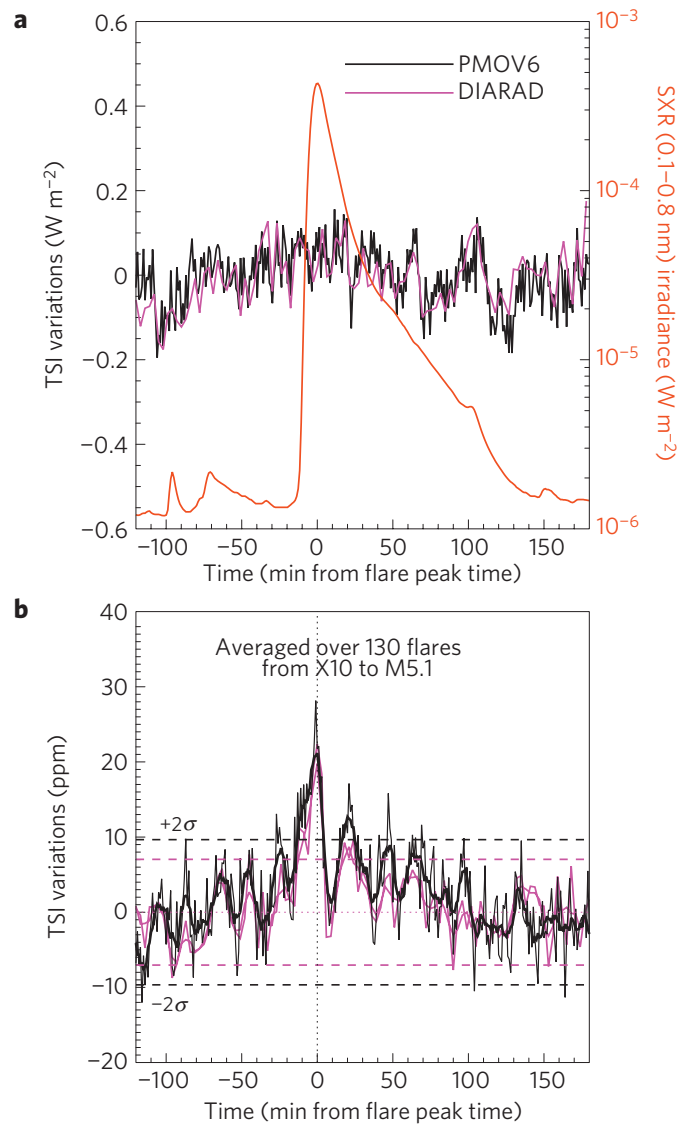

Figure 1 | Detection of flares in the TSI through superposed-epoch analysis. a, Variation of TSI during a single X4 flare, which occurred on 26 November 2000. The flare shows large contrasts in the extreme-ultraviolet and SXR domain but is barely perceptible-if at all-in the TSI. The standard deviation, $\sigma$, of the PMOV 6 time series is $0.07 \mathrm{~W} \mathrm{~m}^{-2}$, which corresponds to about $50 \mathrm{ppm}$ (we assume a mean TSI value of 1,365 to convert from $\mathrm{W} \mathrm{m}^{-2}$ to $\mathrm{ppm}$ ). $\mathbf{b}$, Averaged TSI variations in ppm for 130 of the largest solar flares of the most recent solar cycle occurring at a heliocentric angle $\theta<60^{\circ}$, obtained through a superposed-epoch analysis. Colour-coding is the same as in $\mathbf{a}$. The time step is respectively $3 \mathrm{~min}$ and $1 \mathrm{~min}$ for DIARAD and PMOV6. Thick lines are obtained by averaging over $6 \mathrm{~min}$. The dashed lines correspond to the $95 \%$ confidence levels (twice the standard deviation computed everywhere except for $1 \mathrm{~h}$ around the flare). The averaging reduces the fluctuation level and reveals the coherent contribution of flares.

'LPC2E-Laboratoire de Physique et Chimie de l'Environnement et de l'Espace, UMR 6115 CNRS et Université d'Orléans, 3A Av. de la Recherche Scientifique, Orléans Cedex 2, France, ${ }^{2}$ Physikalisch-Meteorologisches Observatorium Davos and World Radiation Center (PMOD/WRC), Dorfstrasse 33, 7260 Davos Dorf, Switzerland, ${ }^{3}$ Royal Meteorological Institute of Belgium, Ringlaan 3, 1180 Brussels, Belgium, ${ }^{4}$ Solar Influences Data Analysis Centre / Royal Observatory of Belgium, Circular Avenue 3, B-1180 Brussels, Belgium. *e-mail: matthieu.kretzschmar@cnrs-orleans.fr. 

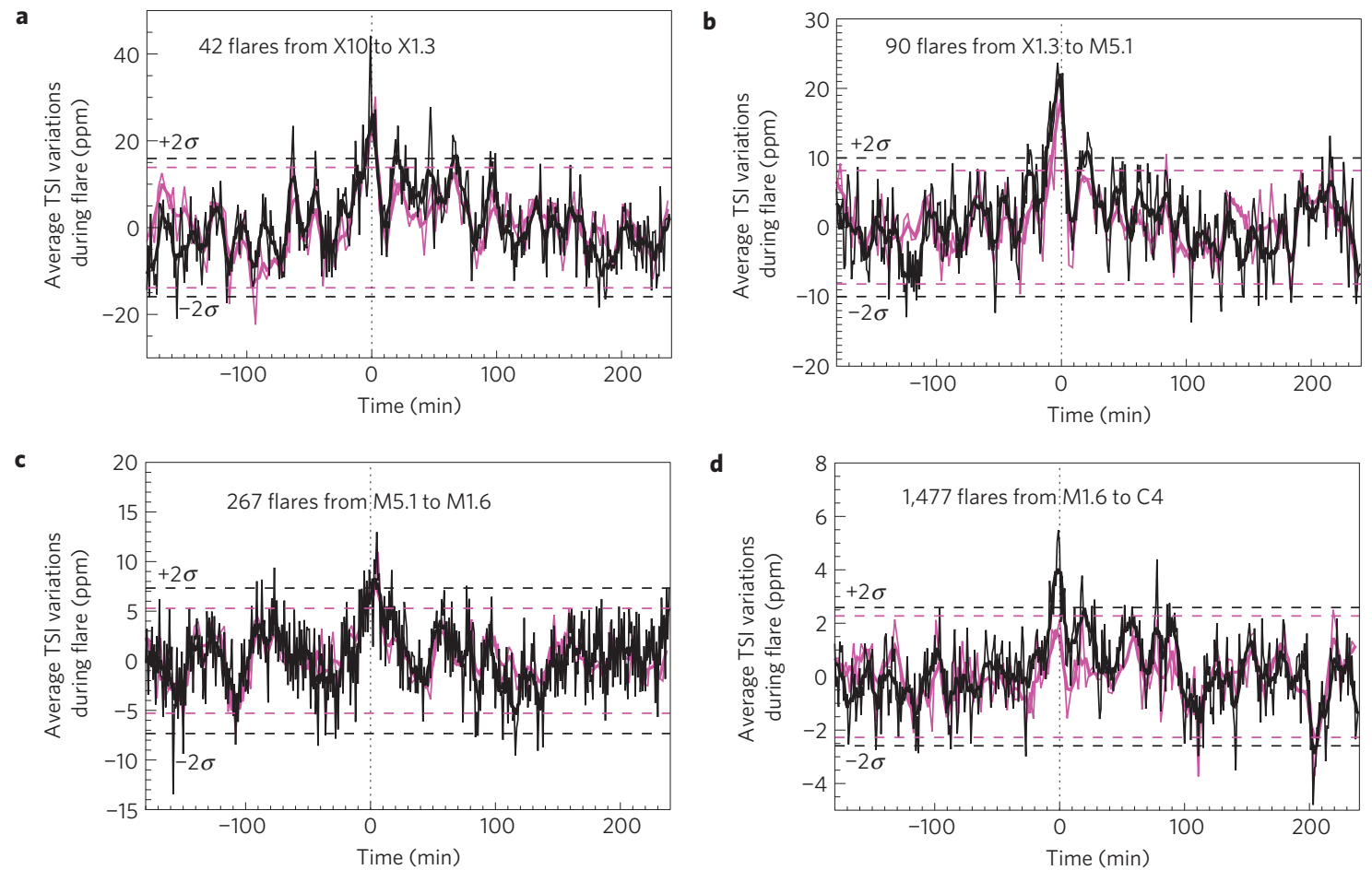

Figure 2 | Averaged TSI variations during flares of smaller amplitudes. a-d, The TSI time-series averages over four exclusive sets of solar flares of decreasing amplitude. The black and pink curves correspond respectively to the TSI measured by the PMOV6 and the DIARAD radiometers. The thick lines are 6 min running averages. The dashed lines correspond to the $95 \%$ confidence levels for the thin curves (twice the standard deviation computed everywhere except for $1 \mathrm{~h}$ around the flare).

We applied this procedure to measurements of the TSI recorded by the PMO and DIARAD radiometers of the VIRGO (ref. 9) experiment onboard the SOHO mission between 1996 and 2007, that is, during the whole 23rd solar cycle. We used the soft-X-ray (SXR) flux observed by the GOES satellites in the spectral range $0.1-0.8 \mathrm{~nm}$ to retrieve the flare information and in particular the peak time. The flare amplitude is given by its $\mathrm{X}$-ray class: the letter indicates a power of ten (' $\mathrm{X}$ ' is $-4,{ }^{\prime} \mathrm{M}$ ' is -5 , ' $\mathrm{C}$ ' is -6 , and so on). A flare of class M4.5 thus corresponds to a SXR peak flux of $I^{\mathrm{SXR}}=4.5 \times 10^{-5} \mathrm{~W} \mathrm{~m}^{-2}$. We considered only flares occurring at heliocentric angle less than $60^{\circ}$ (to limit line-of-sight effects) and of class greater than M3 to stay sufficiently above the instrumental background. To maximize the flare signal, we ranked the flares from the largest to the smallest using the SXR flux peak $F^{\mathrm{SXR}}$ measured by the GOES satellites: $F_{1}{ }^{\mathrm{SXR}}>F_{2}{ }^{\mathrm{SXR}}>\cdots>F_{i}{ }^{\mathrm{SXR}}>\cdots>F_{\mathrm{N}}{ }^{\mathrm{SXR}}$, and we applied the analysis to several sets of flares that are characterized by two values: the largest flare of the set (denoted by its rank $k$ ) and the total number $n$ of flares inside the set (the smallest flare of the set then being of rank $k+n-1)$. For each flare, we selected a TSI interval of $\tau=7 \mathrm{~h}$ in such a way that the flare peak time is situated $180 \mathrm{~min}$ after the interval begins. These short intervals were divided by their time average before being superposed, so the average relative TSI variations resulting from the analysis can be expressed as $\bar{I}(k, n, t)=10^{6} \cdot 1 / n\left(\sum_{i=k}^{k+n-1}\left(I_{i}(t) /\left\langle I_{i}(t)\right\rangle_{\tau}\right)-1\right)$, where $I_{i}(t)$ is the measured TSI time series during the flare of rank $i$ and $\bar{I}(k, n, t)$ is expressed in parts per million (ppm). The analysis is based on the fact that, although the TSI value at peak time $I_{i}\left(t_{\text {peak }}\right)$ cannot be distinguished from the value at other times, it includes a small contribution $I_{i}^{\mathrm{f}}$ due to the flare. At times where no flare emission occurs (far from $t_{\text {peak }}$ ), the incoherent fluctuations are averaged out and the value $I(t)$ goes to zero as $\sigma / \sqrt{n}$, where $\sigma$ is the standard deviation of the original time series. At the peak of the flare, the coherent contribution of the flares causes the average value to differ from zero and to tend towards the average relative
TSI increase $\bar{I}\left(k, n, t_{\text {peak }}\right)=\overline{I^{\mathrm{f}}}(k, n)=1 / n\left(\sum_{i=k}^{k+n-1} I_{i}^{\mathrm{f}}\right)$. This supposes that other phenomena such as acoustic waves and active-region evolution are not in phase with the flares and therefore do not contribute to the flare signature.

We first considered the conditional averaging of 130 very large flares of the most recent solar cycle, shown in Fig. 1b. First it should be noted that the fluctuation level is reduced from its initial level of about $\sigma=50 \mathrm{ppm}$ to about $\sigma(n)=50 / \sqrt{n} \mathrm{ppm}$ in the averaged curve. More importantly, the average TSI clearly reveals a statistically significant peak at the time of the flare. Most of the

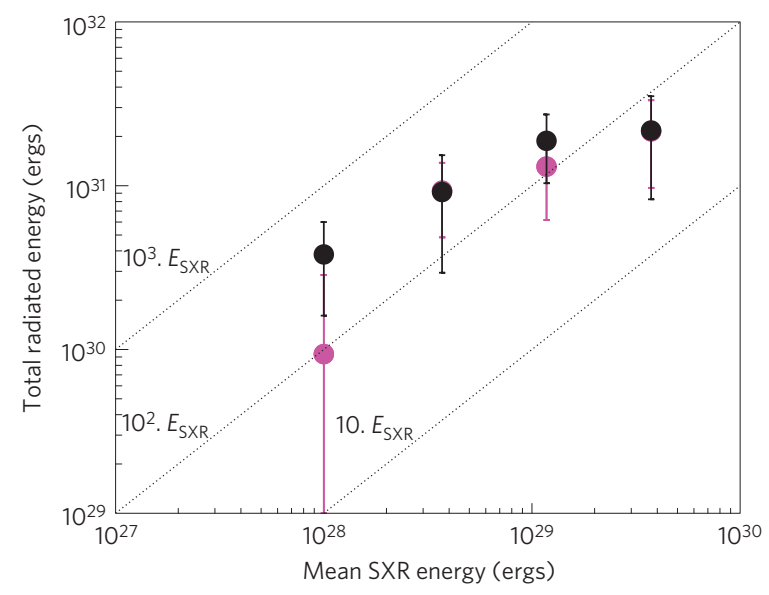

Figure 3 | Total energy radiated by solar flares versus the energy radiated in X-rays. The black and pink symbols correspond respectively to estimates using PMO6V and DIARAD. Each point corresponds to a panel of Fig. 2.

The error bars correspond to the $1 \sigma$ dispersion in the averaged time series and are only indicative; the uncertainties are in fact larger because part of the flare profile is below the noise level. 
TSI increase happens before the key time, which shows that this emission comes mainly from the impulsive phase.

This remarkable result remains valid when we consider flares of lower amplitude (see Fig. 2), for the first time revealing a significant impact on the TSI, not only for the few largest flares but also for the numerous smaller ones. Figure 2 shows four exclusive sets of flares with a clear signature in the TSI at the time of the X-ray flare. For the smallest flare in panel $\mathrm{d}$, the signal is less clear in the DIARAD data, which can at least in part be explained by the smaller duty cycle of the instruments. It is important to note that the apparent periodic patterns in Figs 2 and 1 are due to the random superposition of the pseudo-periodic TSI oscillations at the typical frequency of p-modes $(3 \mathrm{mHz})$; these patterns are more prominent in the smoothed curves but remain below the $2 \sigma$ level, and can thus hardly be interpreted as actual signatures of physical processes related to flares. Figures 1 and 2 show the first important finding of this study, namely that the total radiative output of the Sun is sensitive to both large and small flares.

Finally, let us look at another quantity of great interest: namely the total energy radiated by flares. This energy was simply estimated by integrating the averaged TSI variations over $20 \mathrm{~min}$ around the peak time and multiplying it by the factor $1,365.10^{7 *}(1 \mathrm{AU})^{2 *} 1.4 \pi$, the factor 1.4 being the best compromise between a uniform angular distribution (factor $2 \pi$ ) expected for optically thin wavelengths and a Lambertian distribution (factor $\pi$; ref.7). The fixed integration time avoids errors caused by low signal-to-noise ratio in determining the flare duration.

The main conclusion that can be drawn from the results, which are shown in Fig. 3, is that the total energy radiated by solar flares exceeds by two orders of magnitude the energy radiated in the SXR domain; this scaling holds for two decades of flare amplitudes. This confirms the recent surprisingly large estimate for the largest flares only ${ }^{3,7,10}$, while considerably extending its validity range; this result affects the whole energy budget of flares as well as the relation between flares and TSI. It should also be noted that the values shown in Fig. 3 could actually be underestimated because flares usually emit over a longer time than that used for the integration. The relatively small contribution of X-rays to the total emission points towards a dominant energy contribution of longer wavelengths, mainly in the near-ultraviolet and visible domains. This is supported by extending our analysis to spectral irradiance measurements carried out in the visible domain by the
SPM sensors of the VIRGO instrument, which shows that flares produce an increase in these visible channels that is similar in time and amplitude to that observed in the TSI (not shown). It thus seems that the contributions of the visible and near-ultraviolet emission to the total flare emission are large enough to make the signature of flares detectable in the total radiative output of our star.

Received 5 May 2009; accepted 29 June 2010; published online 15 August 2010

\section{References}

1. Haisch, B., Strong, K. T. \& Rodono, M. Flares on the sun and other stars. Annu. Rev. Astron. Astrophys. 29, 257-324 (1991).

2. Hudson, H. S. \& Willson, R. C. Upper limits on the total radiant energy of solar flares. Sol. Phys. 86, 123-130 (1983).

3. Woods, T. N. et al. Solar irradiance variability during the October 2003 solar storm period. Geophys. Res. Lett. 31, L10802 (2004).

4. Fröhlich, C. \& Lean, J. Solar radiative output and its variability: Evidence and mechanisms. Astron. Astrophys. Rev. 12, 273-320 (2004).

5. Carrington, R. C. Description of a singular appearance seen in the sun on September 1. Mon. Not. R. Astron. Soc. 20, 13-15 (1859).

6. Neidig, D. F. The importance of solar white-light flares. Sol. Phys. 121, 261-269 (1989).

7. Woods, T. N., Kopp, G. \& Chamberlin, P. C. Contributions of the solar ultraviolet irradiance to the total solar irradiance during large flares. J. Geophys. Res. 111, A10S14 (2006).

8. Johnsen, H., Pécseli, H. L. \& Trulsen, J. Conditional eddies in plasma turbulence. Phys. Fluids 30, 2239-2254 (1987).

9. Frohlich, C. et al. First results from VIRGO, the experiment for helioseismology and solar irradiance monitoring on SOHO. Sol. Phys. 170, 1-25 (1997).

10. Emslie, A. G. et al. Refinements to flare energy estimates: A followup to 'Energy partition in two solar flare/CME events'. J. Geophys. Res. 110, 11103 (2005).

\section{Acknowledgements}

This work has received funding from the European Community's Seventh Framework Programme (FP7/2007-2013) under the grant agreement No 218816 (SOTERIA project, www.soteria-space.eu). J-F.H., T.D.d.W. and M.K. also acknowledge financial support from the French-Belgian partnership program 'Tournesol'.

\section{Author contributions}

S.D., S.M., W.S. and J-F.H. were involved in the design of the study. T.D.d.W. was involved in the analysis of the data. M.K. analysed the data and drafted the manuscript. All authors discussed the results and commented on the manuscript.

\section{Additional information}

The authors declare no competing financial interests. Reprints and permissions information is available online at http://npg.nature.com/reprintsandpermissions. Correspondence and requests for materials should be addressed to M.K. 